\title{
Mitochondrial dynamics in cardiovascular disease: fission and fusion foretell form and function
}

\author{
Willard W. Sharp • Stephen L. Archer
}

Received: 3 December 2014 /Revised: 3 December 2014 / Accepted: 26 January 2015 / Published online: 12 February 2015

(C) Springer-Verlag Berlin Heidelberg 2015

Whether it be the sweeping eagle in his flight, or the open apple-blossom, the toiling work-horse, the blithe swan, the branching oak, the winding stream at its base, the drifting clouds, over all the coursing sun, form ever follows function, and this is the law. Where function does not change, form does not change.

Louis Sullivan, The Tall Office Building Artistically Considered, Lippincott's Magazine (March 1896)

Mitochondria are dynamic, semiautonomous, networked, organelles whose architecture is ever changing $[1,2]$. The connectivity of the network, the shape of individual mitochondria and their intracellular location are constantly varying due to processes that, in aggregate, are termed mitochondrial dynamics. The key functions of mitochondria encompassed by the term mitochondrial dynamics are division (fission), union (fusion) and transport (trafficking) [3]. In addition, the cell can create more mitochondria, through mitochondrial biogenesis, and eliminate dysfunctional mitochondria, by mitophagy. Fission results in more numerous, smaller, more isolated mitochondria; conversely, fusion increases the interconnection of mitochondria and permits exchange of mitochondrial matrix proteins and mitochondrial DNA throughout the network. However, more causes and consequences of mitochondrial fission and fusion are being discovered each year. These non-canonical, dynamic, capabilities of mitochondria

W. W. Sharp

Division of Emergency Medicine, Department of Medicine,

University of Chicago, Chicago, IL, USA

S. L. Archer $(\bowtie)$

Department of Medicine, Queen's University, Etherington Hall, Room 3041, 94 Stuart St., Kingston, Ontario, Canada K7L 3N6 e-mail: stephen.archer@queensu.ca are best understood if one views mitochondria as once-independent, prokaryotic organisms that now exist symbiotically in eukaryotic cells [4]. From this perspective, quality control functions that may originally have evolved for benefit of their ancestors (i.e. fusion-mediated complementation of mitochondrial DNA) now offer benefit for the eukaryotic host cell.

While the ability of mitochondria to change shape has been recognized for a century [5], only recently has mitochondrial dynamics emerged as a field of study. In the past decade, substantial progress has been made in defining the underlying molecular mechanisms and identifying the importance of mitochondrial dynamics to cell biology [6]. Acquired disorders of mitochondrial dynamics contribute to the pathogenesis of many common human diseases [1]. Acquired disorders of fission and fusion are likely to be much more prevalent and impactful to the population than the more dramatic (but rarer) monogenic mitochondrial diseases [7]. Mitochondrial dynamics are affected by, and in turn affect, diverse cellular functions including metabolism, proliferation, apoptosis, redox signaling and calcium homeostasis. Acquired disorders of mitochondrial dynamics contribute to the pathophysiology of many cardiovascular diseases, including pulmonary arterial hypertension (PAH), cardiomyopathy and cardiac ischemia reperfusion injury (IR). The mediators of mitochondrial fission and fusion offer a rich array of new therapeutic targets, as will be discussed in this special edition of $J$ Mol Med.

Fission and fusion are accomplished by a relatively small number of mediators, many of which are large guanosine triphosphatases (GTPases). Fission is mediated by dynaminrelated protein 1 (Drp1), a GTPase that resides in the cytosol until activation, whereupon it moves to the outer mitochondrial membrane (OMM). There, Drp1 interacts with binding partners and multimerizes, creating a constricting collar that divides the mitochondria. Fusion is mediated by the large GTPases 
mitofusin-1 and mitofusin-2, in the OMM, and optic atrophy 1 (OPA1), resident in the inner mitochondrial membrane.

Regulation of mitochondrial dynamics occurs at multiple levels, including transcriptional control, alternative splicing and posttranslational modification of the regulatory proteins. Peroxisome proliferator-activated receptor gamma coactivator 1 -alpha $(\mathrm{PGC} 1 \alpha)$ regulation of mitofusin 2 expression is one of the transcriptional regulators of the mitochondrial dynamics pathway [8]. Reduced PGC1 $\alpha$ expression in PAH is a contributing factor to the deficiency of mitofusin-2 that contributes to the hyperproliferative state in PAH, as discussed by Ryan et al. in this special edition [9]. Alternative splicing of the transcripts regulating mitochondrial fission and fusion in specific cell types may also exert tissue and cellular specific control. For example in immune cells, alternative splicing of Drp1 results in the Drp1-x01 isoform that is specifically targeted to the microtubules. This splice variant stabilizes the microtubules, resulting in decreased apoptosis. Phosphorylation of the Drp1-x01 isoform by cyclin-dependent kinase 1 (CDK1) promotes its release from microtubules and leads to fission [10].

However, in most cardiovascular diseases, the predominant regulatory mechanisms of mitochondrial dynamics are posttranslational and include phosphorylation, SUMOylation, ubiquitination, nitrosylation, acetylation and acylation of the fission and fusion mediators. These posttranslational mechanisms alter the activity and/or expression levels of key GTPases. The activity of Drp1, for example, reflects the balance between phosphorylation at Drp1-serine 616 (which promotes activation and translocation to the OMM) versus phosphorylation at Drp1-serine 637, which pacifies Drp1 and prevents fission. These two phosphorylation sites are targets for different kinases and phosphatases. Phosphorylation of Drp1serine 616 is important in coordinating nuclear and mitochondrial division, so-called mitotic fission [11-13]. Although mitochondria are randomly distributed during cell division, the coordination of fission with mitosis is essential to avoid asymmetrical allocation of mitochondria to daughter cells. Drp1serine 616 is phosphorylated by cyclin B-CDK1, a kinase that promotes mitosis initiation $[12,14]$. The shared reliance of nuclear and mitochondrial division on this kinase contributes to the coordination between nuclear and mitochondrial division. After mitosis the Drp1 is ubiquitinated and destroyed allowing the mitochondria to reform into networks. Mitotic fission is emerging as a therapeutic target in proliferative diseases, such as PAH and cancer, as discussed by Ryan et al. in this special issue [9].

In contrast, serine 637 is phosphorylated by protein kinase A (PKA). Dephosphorylation of Drp1-serine 637, by the calcium-sensitive phosphatase, calcineurin, participates in cell death in IR $[15,16]$. Through this mechanism, Drp1 becomes activated in states where intracellular calcium concentrations are pathologically increased, such as cardiac arrest and IR [15]. The ensuing fission-mediated production of reactive oxygen species (ROS) contributes to cardiac dysfunction and can be therapeutically targeted using Drp1 inhibitors, as discussed by Sharp in this issue [17].

Further precision in the regulation of mitochondrial dynamics is provided by organellar interactions that pinpoint the site for assembly of fission mediators. Microdomains on the OMM are formed by interaction with the endoplasmic reticulum [18]. In these microdomains, activated Drp1 is brought into proximity and binds nonGTPase partners, such as Fis1, mitochondrial fragmentation factor 1 (MFF1), Mid49, and Mid51, creating the multimeric fission assembly that divides the organelle [19].

One noncanonical mitochondrial function often affects another. For example, kinesin motors power mitochondrial trafficking on actin filaments to areas of energy need. The mitochondria are attached to the kinesin motors and the microfilaments by the adapter proteins, Miro and Milton. Loss of the mitofusins impairs mitochondrial trafficking by disconnecting the organelle from Miro [20]. Thus, a defect in mitochondrial fusion would be expected to impair mitochondrial trafficking.

As one reads this special edition of $\mathrm{J} \mathrm{Mol} \mathrm{Med}$, we suspect that you will challenge Sullivan's unidirectional assertion that "Where function does not change, form does not change". The architectural analogy is clearly incomplete as applied to the dynamic architecture of the mitochondria. For example, while proliferating cells have fragmented mitochondria because mitosis and mitotic fission are coordinated (form following function), mitochondrial fission in IR drives production of ROS (function following form). This bidirectional relationship between form and function is a reminder that the causes and consequences of mitochondrial dynamics are contextual.

A complexity of mitochondrial dynamics is the observation that mitochondrial morphology rarely has a single molecular basis. For example, increased fragmentation may reflect increased fission or decreased fusion (or both). Moreover, the underlying molecular mechanism (activated Drp1 versus deficient mitofusin-2) cannot be inferred by pattern recognition but must instead be measured. Even once the expression of crucial mediators is established, the ubiquitous role of posttranslational modification must be considered. It is also challenging to determine whether a particular mitochondrial morphology is physiologic or pathologic, since a single phenotype (fragmentation) can be normal (e.g. mitotic fission in a dividing cell) or pathologic (e.g. IR-induced fission in an infarcting cardiomyocyte). There is also substantial structural diversity among mitochondria in different cell types. For example, adult cardiomyocytes have tightly compartmentalized mitochondria and this ultrastructure minimizes organelle movement whereas in vascular cells and neurons mitochondrial trafficking, fission and fusion are prominent.

Temporal and quantitative considerations are also key in understanding mitochondrial dynamics, and tools are required to quantify rates of mitochondrial turnover and define 
mitochondrial structure-function relationships. For example, molecular deletion of a fission or fusion mediator in a healthy organism (e.g. the Drp1-knockout mouse) does not always have the same effect as transient, partial inhibition of the same mediator during a pathologic challenge. Sharp in this issue [17] contrasts the cardiotoxic effects of Drp1 knockout in mice versus the cardioprotective effects of specifically inhibiting Drp1's GTPase activity in rats undergoing myocardial IR injury using pharmacological agents, such as Mdivi-1 or P110. The mouse knockout model results in tonic, complete loss of Drp1 in an otherwise healthy mouse; the pharmacological intervention results in transient, partial inhibition of an activated fission pathway.

Fission and fusion may occur in coordination to achieve a single cellular function. For example, fission and fusion collaborate to allow critical quality control functions, such as mitophagy. Mitochondrial fusion can dilute protein oxidative damage and the accumulation of mitochondrial DNA mutations through a process called complementation. However, when the degree of heteroplasmy or oxidative damage is excessive, mitochondrial fission asymmetrically divides the organelle allowing mitophagic elimination of the depolarized portion of the mitochondria. These depolarized, mitofusin-deficient, portions of the organelle are excluded from the network. They become targets for Parkin-mediated ubiquitination which marks them for elimination by mitophagy [21, 22]. Thus both fusion and fission are (at the right time and in the right amount) part of the mitochondrial quality control mechanisms, as discussed by Shires and Gustafsson in this edition [23].

Issue outline In the first three articles of this issue, the role of mitochondrial dynamics in pulmonary hypertension, cardiac arrest and heart failure are specifically discussed. We begin with Ryan et al. who examine the role of mitochondrial dynamics in pulmonary hypertension [9]. Mitochondrial division is increasingly recognized as a potential checkpoint for cell cycle progression and abnormalities in mitochondrial dynamics have been implicated in pathologies involving abnormal cellular proliferation such as cancer and pulmonary hypertension. The role of mitochondrial fission and fusion in pulmonary hypertension and the value of mitofusin-2 and Drp1 as targets of therapy of this disease are reviewed. The next article, by Sharp, examines the role of Drp1 in myocardial dysfunction following cardiac arrest [17]. Posttranslational modifications of Drp1 following cardiac IR result in Drp1's translocation to the mitochondria and fission-induced ROS production. The resulting impairment of calcium handling causes myocardial diastolic dysfunction. Administration of Drp1 inhibitors early during reperfusion are cardioprotective and improve outcomes. Next, Shires and Gustafsson discuss the role of mitochondrial quality control in the setting of heart failure [23]. Mitochondrial quality control is important for cellular health and proper functioning of the heart. Disturbing the balance of mitochondrial dynamics impairs the mitophagic elimination of damaged mitochondria and contributes to progressive injury of the myocardium, promoting heart failure.

The next three articles discuss the role of mitochondrial lipids, innovative mitochondrial imaging techniques, and pharmacological therapies for manipulating mitochondrial dynamics. Frohman reminds us that mitochondrial fission can occur independently of changes in Drp1 activity, regulated instead by modification of mitochondrial lipids themselves [24]. Harkening their origins as prokaryotic bacteria, the mitochondrial membranes are unique sources of cardiolipin, a lipid otherwise found only in bacteria. Cardiolipin and its metabolites, phosphatidic acid and lysophosphatidic acid, modulate fusion and fission, as discussed by Frohman in this special edition [24]. Gottlieb next describes innovative techniques of tracking and imaging mitochondrial turnover with Mitotimer both in vivo and in vitro [25]. Finally, an examination of new mitochondrial therapeutic targets by Disatnik et al. is presented [26]. We believe these articles taken together will provide the reader with an overview of the importance of mitochondrial dynamics in cardiovascular disease.

\section{Conclusion}

Changes in the balance between fission and fusion rates have downstream effects on rates of proliferation and apoptosis [11, 12], metabolism [27] and the production of reactive oxygen species (ROS) [28]. Drp1 is involved in process as diverse as cell division [12, 13], bioenergetics [29], apoptosis and physiologic ROS signaling [28]. Likewise, fusion is also critical in regulating cell proliferation and bioenergetics [27]. These non-canonical, dynamic properties of mitochondria recast this organelle as much more than a source of energy [3, 30]. As elegantly proposed by the architect Sullivan, form follows function; however, it is now clear that mitochondrial form also foretells mitochondrial function.

Acknowledgements This work is supported by NIH K08 HL10390101A1 and RO3 HL110826-01A1 (WS) and NIH-RO1-HL071115 and 1RC1HL099462 (SLA).

Disclosure None

\section{References}

1. Archer SL (2013) Mitochondrial dynamics-mitochondrial fission and fusion in human diseases. N Engl J Med 369:2236-2251

2. Westermann B (2010) Mitochondrial fusion and fission in cell life and death. Nat Rev Mol Cell Biol 11:872-884

3. Youle RJ, van der Bliek AM (2012) Mitochondrial fission, fusion, and stress. Science 337:1062-1065 
4. Margulis L (1975) Symbiotic theory of the origin of eukaryotic organelles; criteria for proof. Symp Soc Exp Biol 21-38

5. Lewis MR, Lewis WH (1914) Mitochondria in tissue culture. Science 39:330-333

6. Kasahara A, Scorrano L (2014) Mitochondria: from cell death executioners to regulators of cell differentiation. Trends Cell Biol 24: 761-770

7. Koopman WJ, Willems PH, Smeitink JA (2012) Monogenic mitochondrial disorders. N Engl J Med 366:1132-1141

8. Ryan JJ, Marsboom G, Fang YH, Toth PT, Morrow E, Luo N, Piao L, Hong Z, Ericson K, Zhang HJ et al (2013) PGClalpha-mediated mitofusin-2 deficiency in female rats and humans with pulmonary arterial hypertension. Am J Respir Crit Care Med 187:865-878

9. Ryan JJ, Dasgupta A, Huston J, Chen KH, Archer SL (2014) Mitochondrial dynamics in pulmonary arterial hypertension. J Mol Med. doi:10.1007/s00109-015-1263-5

10. Strack S, Wilson TJ, Cribbs JT (2013) Cyclin-dependent kinases regulate splice-specific targeting of dynamin-related protein 1 to microtubules. J Cell Biol 201:1037-1051

11. Marsboom G, Toth PT, Ryan JJ, Hong Z, Wu X, Fang YH, Thenappan T, Piao L, Zhang HJ, Pogoriler J et al (2012) Dynamin-related protein 1-mediated mitochondrial mitotic fission permits hyperproliferation of vascular smooth muscle cells and offers a novel therapeutic target in pulmonary hypertension. Circ Res 110:1484-1497

12. Rehman J, Zhang HJ, Toth PT, Zhang Y, Marsboom G, Hong Z, Salgia R, Husain AN, Wietholt C, Archer SL (2012) Inhibition of mitochondrial fission prevents cell cycle progression in lung cancer. FASEB J 26:2175-2186

13. Taguchi N, Ishihara N, Jofuku A, Oka T, Mihara K (2007) Mitotic phosphorylation of dynamin-related GTPase Drp1 participates in mitochondrial fission. J Biol Chem 282:11521-11529

14. Mitra K, Wunder C, Roysam B, Lin G, Lippincott-Schwartz J (2009) A hyperfused mitochondrial state achieved at G1-S regulates cyclin E buildup and entry into S phase. Proc Natl Acad Sci U S A 106: 11960-11965

15. Sharp WW, Fang YH, Han M, Zhang HJ, Hong Z, Banathy A, Morrow E, Ryan JJ, Archer SL (2014) Dynamin-related protein 1 (Drp1)-mediated diastolic dysfunction in myocardial ischemiareperfusion injury: therapeutic benefits of Drp1 inhibition to reduce mitochondrial fission. FASEB J 28:316-326

16. Cribbs JT, Strack S (2009) Functional characterization of phosphorylation sites in dynamin-related protein 1. Methods Enzymol 457: 231-253
17. Sharp WW (2014) Dynamin related protein 1 (Drp1) as a therapeutic target in cardiac arrest. J Mol Med. doi:10.1007/s00109-015-1257-3

18. Lackner LL, Ping H, Graef M, Murley A, Nunnari J (2013) Endoplasmic reticulum-associated mitochondria-cortex tether functions in the distribution and inheritance of mitochondria. Proc Natl Acad Sci U S A 110:E458-E467

19. Loson OC, Song Z, Chen H, Chan DC (2013) Fis1, Mff, MiD49, and MiD51 mediate Drp1 recruitment in mitochondrial fission. Mol Biol Cell 24:659-667

20. Misko A, Jiang S, Wegorzewska I, Milbrandt J, Baloh RH (2010) Mitofusin 2 is necessary for transport of axonal mitochondria and interacts with the Miro/Milton complex. J Neurosci 30:4232-4240

21. Twig G, Shirihai OS (2011) The interplay between mitochondrial dynamics and mitophagy. Antioxid Redox Signal 14:1939-1951

22. Geisler S, Holmstrom KM, Treis A, Skujat D, Weber SS, Fiesel FC, Kahle PJ, Springer W (2010) The PINK1/Parkin-mediated mitophagy is compromised by $\mathrm{PD}$-associated mutations. Autophagy 6:871-878

23. Shires S, Gustafsson A (2014) Mitochondrial quality control: the difference between success and heart failure. J Mol Med. doi:10.1007/s00109-015-1254-6

24. Frohman M (2014) Role of mitochondrial lipids in guiding fission and fusion. J Mol Med. doi:10.1007/s00109-014-1237-z

25. Gottlieb RA, Stotland A (2014) MitoTimer: a novel protein for monitoring mitochondrial turnover. J Mol Med. doi:10.1007/s00109-0141230-6

26. Disatnik M-H, Ferreira JCB, Hwang S, Mochly-Rosen D (2014) New therapeutics to modulate mitochondrial dynamics and mitophagy in cardiovascular diseases. J Mol Med. doi:10.1007/ s00109-015-1256-4

27. Chen H, Chomyn A, Chan DC (2005) Disruption of fusion results in mitochondrial heterogeneity and dysfunction. J Biol Chem 280: 26185-26192

28. Hong Z, Kutty S, Toth PT, Marsboom G, Hammel JM, Chamberlain C, Ryan JJ, Zhang HJ, Sharp WW, Morrow E et al (2013) Role of dynamin-related protein 1 (Drp1)-mediated mitochondrial fission in oxygen sensing and constriction of the ductus arteriosus. Circ Res 112:802-815

29. Benard G, Bellance N, James D, Parrone P, Fernandez H, Letellier T, Rossignol R (2007) Mitochondrial bioenergetics and structural network organization. J Cell Sci 120:838-848

30. Lee YJ, Jeong SY, Karbowski M, Smith CL, Youle RJ (2004) Roles of the mammalian mitochondrial fission and fusion mediators Fis1, Drp1, and Opa1 in apoptosis. Mol Biol Cell 15:5001-5011 